\title{
Cadmium-induced adrenal cortical autophagy in rats: possible modulation by sildenafil
}

\author{
R.A. Imam, A.G. Motawei, W.A. Abd Algaleel \\ Department of Anatomy and Embryology, Faculty of Medicine, Cairo University, Cairo, Egypt \\ [Received: 7 August 2019; Accepted: 3 November 2019]
}

Background: The link between autophagy, inflammatory bowel disease, ischaemic injury and cancer had been established. Reasonable evidence is available for cadmium to be related to certain cancers. Sildenafil had been investigated to modulate oxidative stress mechanisms. The aim of this study is to investigate cadmium-induced adrenal cortical autophagy and to declare possible modulation by sildenafil.

Materials and methods: Twenty four Wistar rats weighing 150-200 g were randomly and equally assigned into: control group, sildenafil ( $20 \mathrm{mg} / \mathrm{kg} /$ day orally) exposed group, cadmium group (cadmium chloride $1 \mathrm{mg} / \mathrm{kg} /$ day SC), cadmium + sildenafil group (rats received cadmium concomitant with sildenafil). Euthanasia was done 4 weeks from the beginning of experiment; adrenal glands were subjected to biochemical, histological, ultrastructural and immunnohistochemical assessment.

Results: Control and sildenafil exposed groups exhibited nearly similar results. Cadmium had produced adrenal cortical apoptosis and ultrastructural derangement of cell organelles. Cadmium-induced autophagy was detected by ultrastructural abundance of enlarged lysosomes and significant $(p<0.05)$ increase in the optical density of lysosomal associated membrane protein 2 immunoexpression. Sildenafil taken with cadmium had decreased adrenal cortical autophagy, significantly modulated the adrenal gland superoxide dismutase and malondialdehyde compared to cadmium group. Also, the optical density of nuclear factor kappa $B$ (NF- $\kappa B$ ) and caspase-3 immunoexpression was significantly decreased in cadmium + sildenafil compared to cadmium group.

Conclusions: Cadmium might induce adrenal cortical autophagy in rats and sildenafil might show an ameliorating effect probably through enhancement of antioxidant defence mechanism and modulation of NF- $\kappa$ B. (Folia Morphol 2020; 79, 4: 709-719)

Key words: autophagy, cadmium, adrenal, sildenafil rats

\section{INTRODUCTION}

Cadmium is found in solders, used in batteries and as alloy in metal platings and coatings. Reasonable evidence is available for cadmium to be associated with prostatic cancer [15]. Oxidative stress-mediated cell death and oxygen free radicals production had been suggested to mediate cadmium-induced cellular toxicity [22].

In ischaemic injury and some types of myopathies, extensive autophagy was noticed. Also, in inflammatory bowel disease, polymorphisms in a gene involved in autophagy have been associated. Furthermore, the 
link between autophagy and cancer had been established [16]. Autophagy, a shuttling of senescent organelles and large protein complexes into lysosomes, is a survival mechanism in times of nutrient deprivation, by which the starved cell can live by digesting its own contents and recycling these contents to provide nutrients and energy $[13,16]$. Autophagy might progress to cell death if the stimulus is more severe despite being a mean of avoiding cell death, or the cell metabolic pathways may switch to apoptosis [4].

Sildenafil is a competitive reversible inhibitor of phosphodiesterase 5 (PDE5) that prevents the catabolism of cyclic guanosine monophosphate (cGMP), prolonging its actions and promoting vasodilation increasing the blood flow to the penis, leading to an erection. Sildenafil is the only agent of PDE5 inhibitors currently approved for the treatment of pulmonary hypertension [27]. In rat models of stroke, sildenafil had been reported to increase brain levels of cGMP. Furthermore, it enhanced the cerebral blood flow level in the hypoperfused region, increased angiogenesis and neurogenesis which can rapidly enhance functional recovery $[17,30]$. High dose of sildenafil had been proved to protect against carbon tetrachloride-induced adverse renal changes by modulation of redox homeostasis in rats [1]. The current study was carried out to investigate cadmium-induced adrenal cortical autophagy and to declare possible modulation by sildenafil.

\section{MATERIALS AND METHODS}

\section{Animals}

The current work followed all ethics of animal research and was approved by the Institutional Animal Care and Use Committee of Cairo University (CU-IACUC) No CU/III/F/39/19. The present study was carried out on 24 male Wistar rats weighing 150-200 g, housed in the Animal House, Faculty of Medicine, Cairo University under standard laboratory and environmental conditions with free access to food and water at a temperature of $\left(20 \pm 2^{\circ} \mathrm{C}\right)$ with a natural 12-h light/dark cycle.

\section{Chemicals}

Cadmium. Cadmiun chloride salt was purchased from Sigma Chemical Co. (St. Luis, Missouri, USA). After dissolving in saline, it was offered every day by $\mathrm{SC}$ injection (1 $\mathrm{mg} / \mathrm{kg} /$ day) [2].

Sildenafil. Sildenafil tablets (Viagra $50 \mathrm{mg}$, Pfizer, Egypt) were dissolved in $10 \mathrm{~mL}$ saline and given at a dose of $(20 \mathrm{mg} / \mathrm{kg})$ offered every day by oral gavage [1].

\section{Experimental design}

The rats were randomly assigned into four equal groups (6 rats each) as follows: control group (received oral saline), sildenafil exposed group (received oral sildenafil), cadmium group (received cadmium), cadmium + sildenafil group (received cadmium concomitant with sildenafil).

All animals were weighed just before sacrification. They were euthanized after 4 weeks from the beginning of the experiment by Pentobarbital $150 \mathrm{mg} / \mathrm{kg}$ IP. After euthanasia of animals, the two adrenal glands of each animal were immediately removed, weighed, half of one adrenal was used for biochemical assessment, the other half underwent paraffin blocking for histological evaluation. The other adrenal gland had undergone electron microscopy processing.

\section{Biochemical analysis}

Adrenal gland homogenates were centrifuged at 4000 r.p.m. for $20 \mathrm{~min}$ and refrigerated at $4^{\circ} \mathrm{C}$. The supernatant was used for estimation of the quantitative activities of and malondialdehyde (MDA) and superoxide dismutase (SOD) as described in Adeyanju et al. 2018 [1].

\section{Histological and immunohistochemical study}

Adrenal gland sections of $5 \mu \mathrm{m}$ thickness were haematoxylin and eosin (H\&E) stained. Immunohistochemical study [25], utilising Dako automated technique, was done to deparaffinised sections for staining with lysosomal associated membrane protein 2 (LAMP2), nuclear factor kappa B (NF- $\kappa$ B), and caspase-3. Primary antibodies used were LAMP2 (Rabbit polyclonal, Genetex, USA), NF- $\kappa$ B p65 (Rabbit polyclonal, ThermoFisher, USA), caspase-3 (Rabbit polyclonal, Santa Cruz, USA). Dako EnVision Flex/HRP was utilised as secondary antibody and Leica ICC50 microscope was used for photographing all histological and immunosistochemical sections.

For ultrastructural study [12], adrenal glands were fixed for 2 hours in $2.5 \%$ glutaraldehyde buffered with $0.1 \mathrm{M}$ cacodylate at $\mathrm{pH} 7.2,1 \%$ osmium tetroxide postfixed, alcohol dehydrated, embedded in epoxy resin mixture after immersion in propylene oxide. Semi-thin sections of about $1 \mu \mathrm{m}$ thickness $1 \%$ toluidine blue stained, and then photographed with Leica ICC50 light microscope. Ultrathin sections ( $80-90 \mathrm{~nm}$ ) were obtained using an LKB ultratome, and stained by uranyl acetate and lead citrate. The ultrastructural photographing was done using 
Table 1. Comparison of the mean body and adrenal gland weight among the different groups

\begin{tabular}{lcc}
\hline Groups & $\begin{array}{c}\text { Mean body weight [g] } \\
\text { (No of animals in each group = 6) }\end{array}$ & $\begin{array}{c}\text { Mean adrenal gland weight [g] } \\
\text { (No of animals in each group = 6) }\end{array}$ \\
\hline Control & $200.5 \pm 11.4$ & $0.028 \pm 0.004$ \\
Sildenafil & $200.8 \pm 16.0$ & $0.027 \pm 0.004$ \\
Cadmium & $177.0 \pm 12.4^{*}, \#$ & $0.026 \pm 0.006$ \\
Cadmium + sildenafil & $191.7 \pm 13.9$ & $0.027 \pm 0.005$ \\
\hline
\end{tabular}

*,${ }^{*}$ - statistically significant compared to control, sildenafil exposed, cadmium groups respectively

a transmission electron microscope (Joel Jem 1400, Germany), Faculty of Agriculture, Cairo University, Egypt.

\section{Histomorphometric study}

The software Leica Quin 500, Germany was utilised to assess the optical density of LAMP2, NF- $\kappa$ B and caspase-3 immuno-reaction in a standard measuring frame using a magnification $\times 400$. Values were presented as a mean and standard deviation (SD) and statistically analysed.

\section{Statistical analysis}

Morphometric, biochemical and histomorphometric data were statistically analysed utilising SPSS version 25.0 (IBM Corporation, Somers, NY, USA) statistical software. They were expressed as means \pm SD. One-way analysis of variance (ANOVA) had been performed, then post-hoc Tukey test was utilised to compare between groups.

\section{RESULTS}

\section{Clinical data, body and adrenal gland weight}

No mortality had been reported among rats. The body weight of cadmium group was significantly decreased compared to the control group. No significant difference ( $p>0.05)$ among the groups was noticed in adrenal gland weight (Table 1).

\section{Biochemical results}

Malondialdehyde of cadmium group was significantly higher (238\%) than that of control group. MDA of cadmium + sildenafil was non-significantly higher (61\%) than that of control group, but was significantly lower (52\%) than that of cadmium group. SOD of cadmium group was significantly lower $(66 \%)$ than that of control group. SOD of cadmium + sildenafil was significantly lower $(33 \%)$ than that of control group, but significantly higher (33\%) than that of cadmium group (Fig. 1).

\section{Histological results}

By H\&E (Figs. 2, 3) staining, control and sildenafil groups exhibited regular cells in all zones with dilated sinusoids in Zona Reticularis (ZR) of sildenafil group. Cadmium group showed disturbed architecture, wide inercellular spaces, cytoplasmic vaculations and frequent apoptosis as well karyolysis. Cadmium + sildenafil group showed slight vaculation in Zona Glomerulosa (ZG), minimal apoptosis in Zona Fasciculata (ZF) and lipofuscin pigments in ZR. Toluidine blue staining (Fig. 4) of the adrenal cortex of the control and sildenafil groups was similar. Cadmium group ZG and ZF sowed many large lipid droplets compressing neighbouring nuclei while cadmium ZR revealed cytoplasmic rarefaction with marked nuclear pleomorphism. Cadmium + sildenafil group exhibited slight nuclear degeneration in ZF.

\section{Electron microscopy results}

Control and sildenafil groups exhibited similar findings. Cadmium group ZG cells appeared with dilated smooth endoplasmic reticulum while cadmium ZF spongiocytes and ZR cells exhibited shrunken nuclei, dilatation of smooth endoplasmic reticulum, loss of mitochondrial cristae and perinuclar swelling as well as abundant enlarged lysosomes containing many digested organelles. Cadmium + sildenafil group revealed cells with intact mitochondria, many lipid droplets and few lysosomes in ZF (Figs. 5, 6).

\section{Immunohistochemical results}

By LAMP2 immunostaining (Fig. 7), control and sildenafil groups exhibited similar results with mild reaction in all zones of the cortex. Cadmium group showed markedly increased LAMP2 while cadmium + sildenafil group showed mild to moderate reaction.

By NF- $\kappa$ B immunostaining (Fig. 8), control and sildenafil groups exhibited similar results with mild reaction in all zones of the cortex. Cadmium group showed strong NF- $\kappa \mathrm{B}$ immunoexpression in all zones 

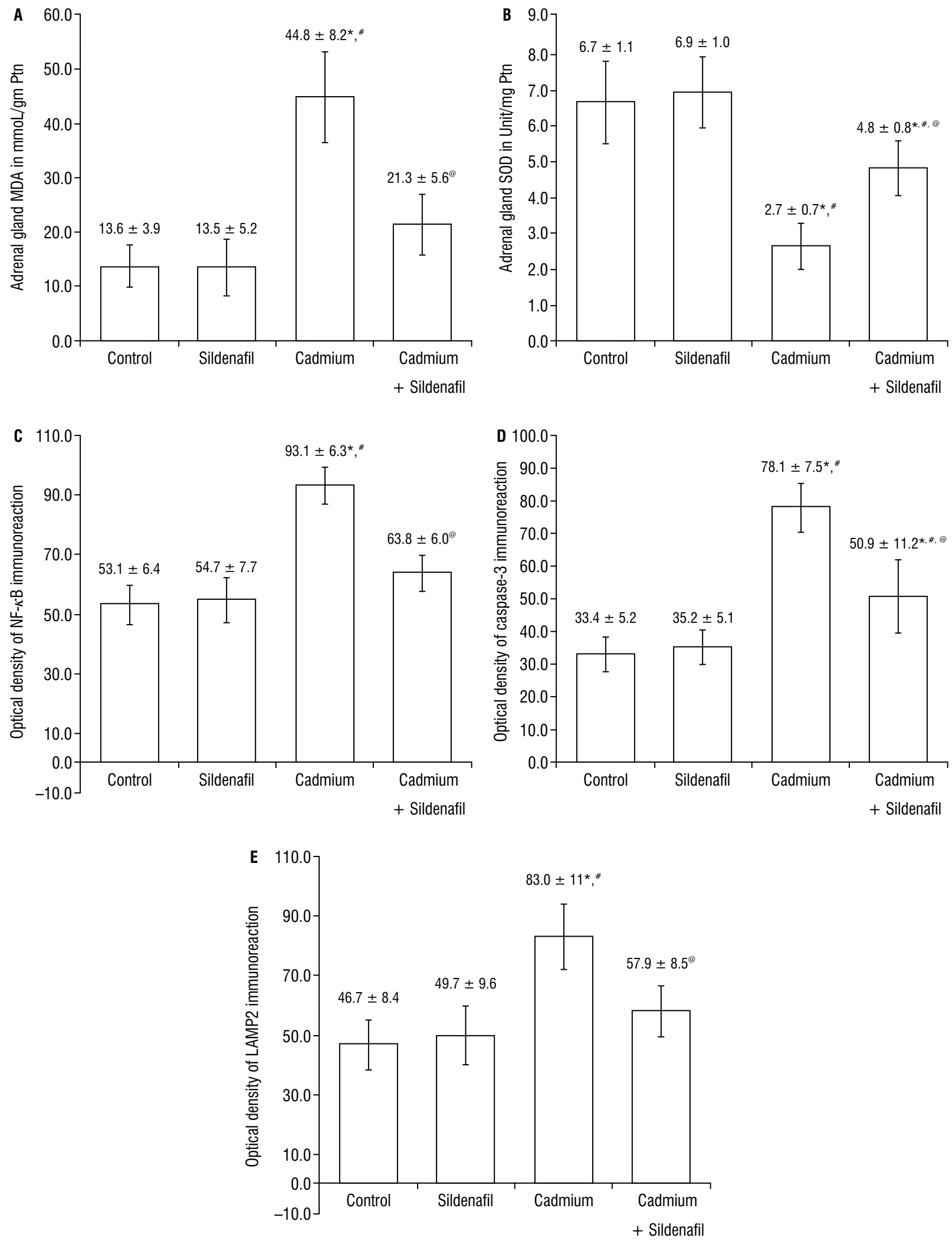

Figure 1. Charts of the mean of adrenal gland malondialdehyde (MDA; $\mathbf{A})$ and superoxide dismutase (SOD; $\mathbf{B})$ as well as the optical density of lysosomal associated membrane protein 2 (LAMP2; E), nuclear factor kappa B (NF- $\kappa$ B; C) and caspase-3 immunoreaction (D) among different groups (no of each group $=6$ ); ${ }^{*}$, ,, - statistically significant compared to control, sildenafil exposed, cadmium groups, respectively. 



Figure 2. Control and sildenafil groups are showing regular cells in all zones with dilated sinusoids sinusoids (S) in Zona Reticularis of sildenafil group $(H \& E \times 400)$
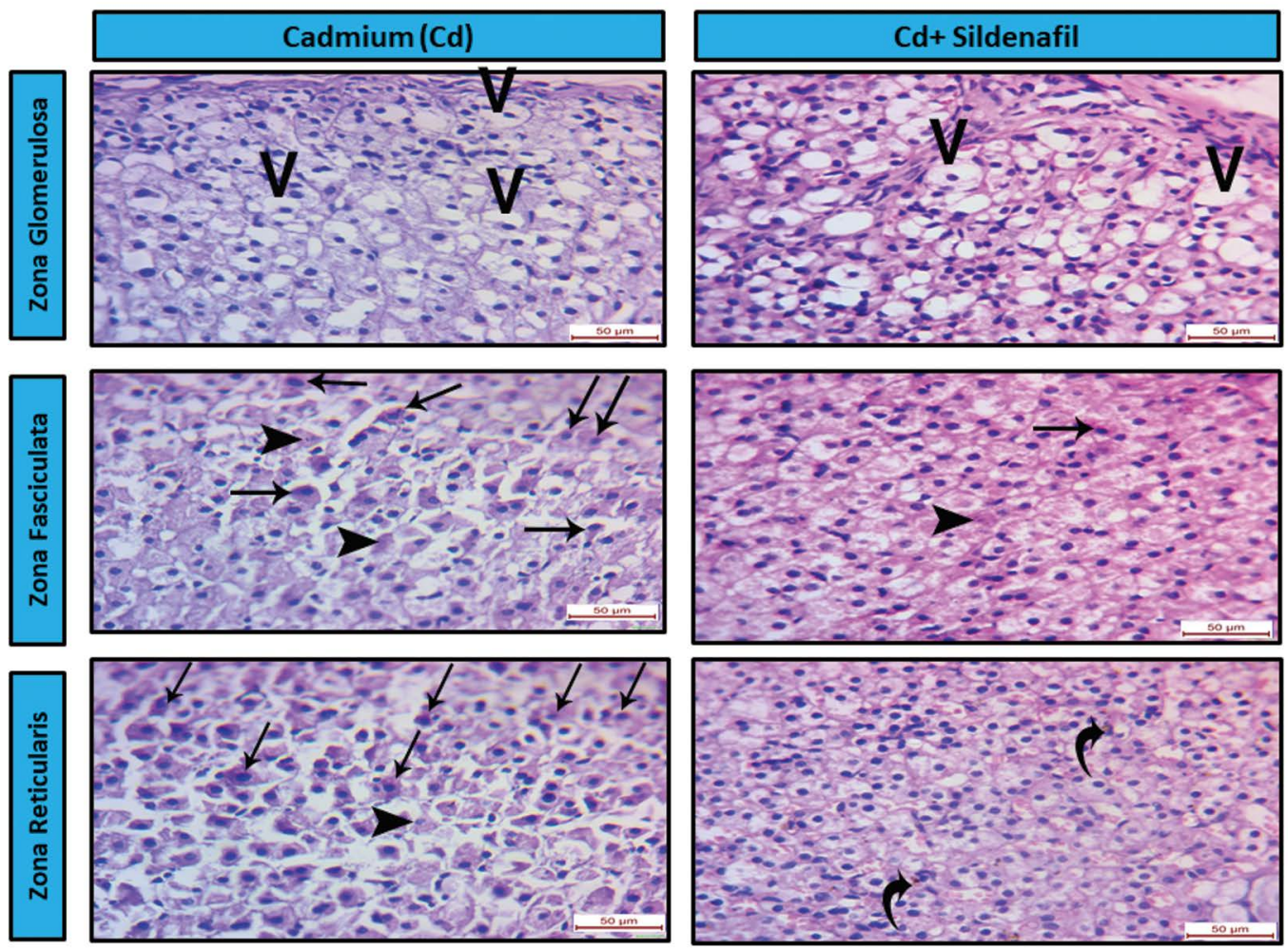

Figure 3. Cadmium group is showing frequent apoptosis (arrows), frequent karyolysis (arrow heads), disturbed architecture, wide inercellular spaces and numerous cytoplasmic vaculations (V). Cadmium + sildenafil group is showing slight vaculation in Zona Glomerulosa, minimal apoptosis in Zona Fasciculata and lipofuscin (curved arrows) pigments in Zona Reticularis (H\&E $\times 400)$. 

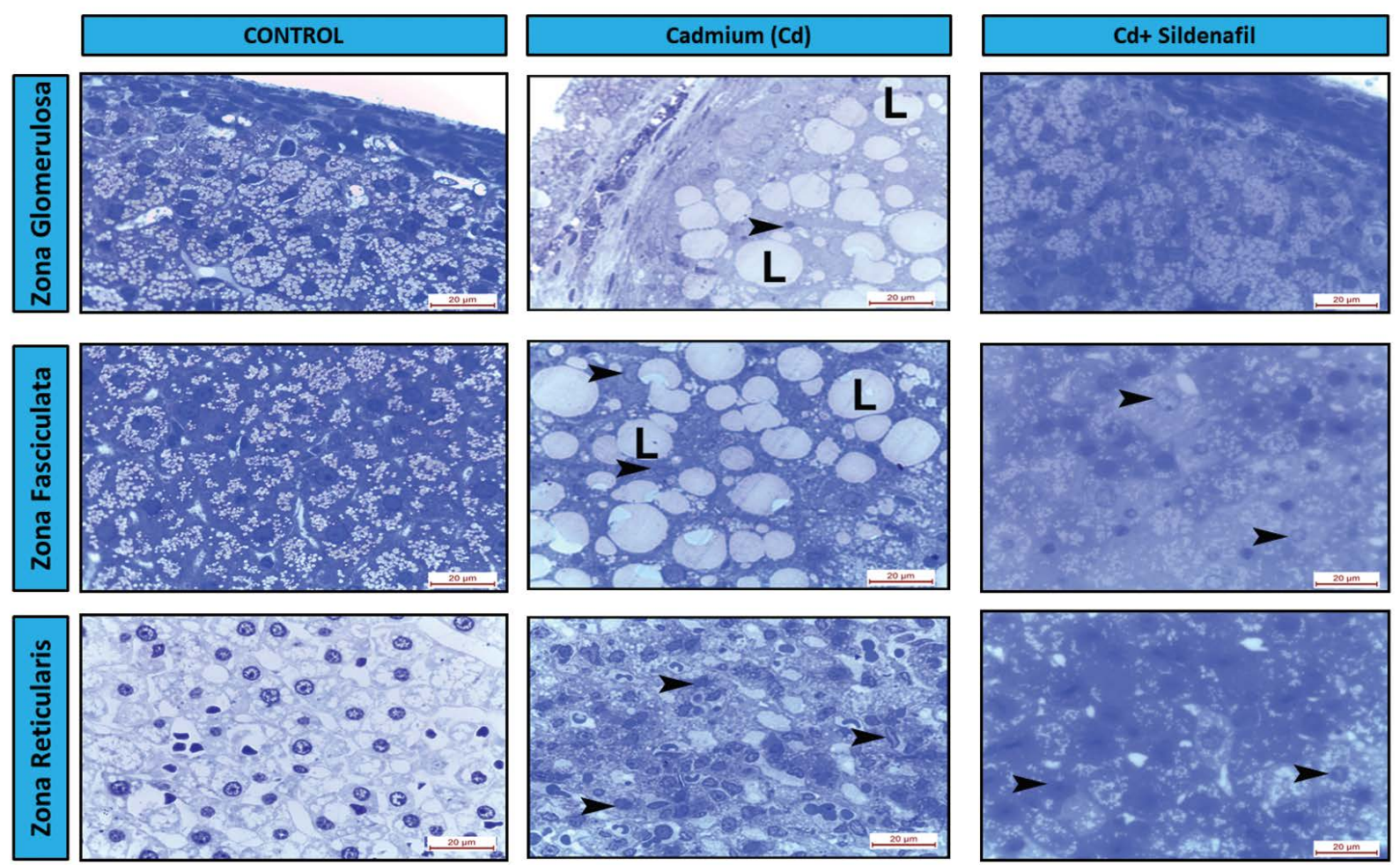

Figure 4. Cadmium group Zona Glomerulosa and Zona Fasciculata (ZF) are exhibiting many large lipid droplets (L) compressing neighbouring nuclei (arrow head) while cadmium Zona Reticularis is exhibiting cytoplasmic rarefaction with marked nuclear (arrow heads) pleomorphism. Cadmium + sildenafil group is showing slight nuclear degeneration in ZF (Toluidine blue $\times 1000$ ).


Figure 5. Cadmium group Zona Glomerulosa cells is appearing with dilated smooth endoplasmic reticulum while cadmium Zona Fasciculata (ZF) and Zona Reticularis cells are exhibiting shrunken nuclei with dilatation of smooth endoplasmic reticulum, loss of mitochondrial (M) cristae as well as abundant enlarged lysosomes (LY) containing digested organelles. Cadmium + sildenafil group appear with many lipid droplets (L) and few lysosomes in ZF (Electron microscopy). 

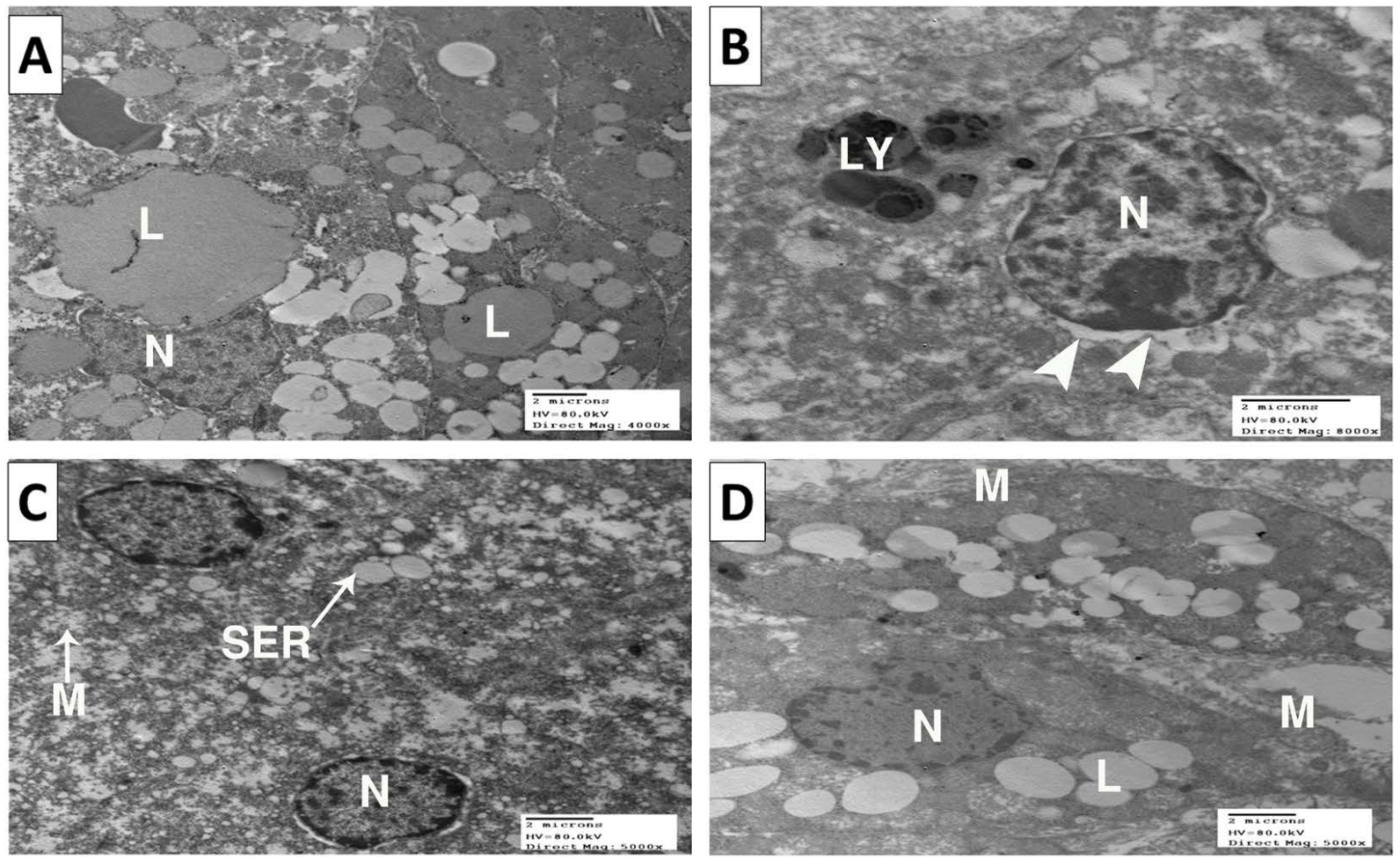

Figure 6. A-C. Cadmium group Zona Glomerulosa, Zona Fasciculata (ZF) and Zona Reticularis, respectively, are showing large lipid droplets (L) compressing the nucleus, dilatation of smooth endoplasmic reticulum (SER), abundant lysosomes (LY), perinuclar swelling (arrow heads) and mitochondrial (M) degeneration. D. Cadmium + sildenafil group ZF appears with abundant mitochondria and lipid droplets (Electron microscopy).


Figure 7. Cadmium group is showing strong lysosomal associated membrane protein 2 (LAMP2) (arrows) immunoexpression while cadmium + sildenafil group is showing mild to moderate reaction (LAMP2 $\times 400)$. 



Figure 8. Cadmium group is showing strong nuclear factor kappa $B(N F-\kappa B)$ (arrows) immunoexpression while cadmium + sildenafil group is showing moderate reaction (NF- $\kappa \mathrm{B} \times 400$ ).
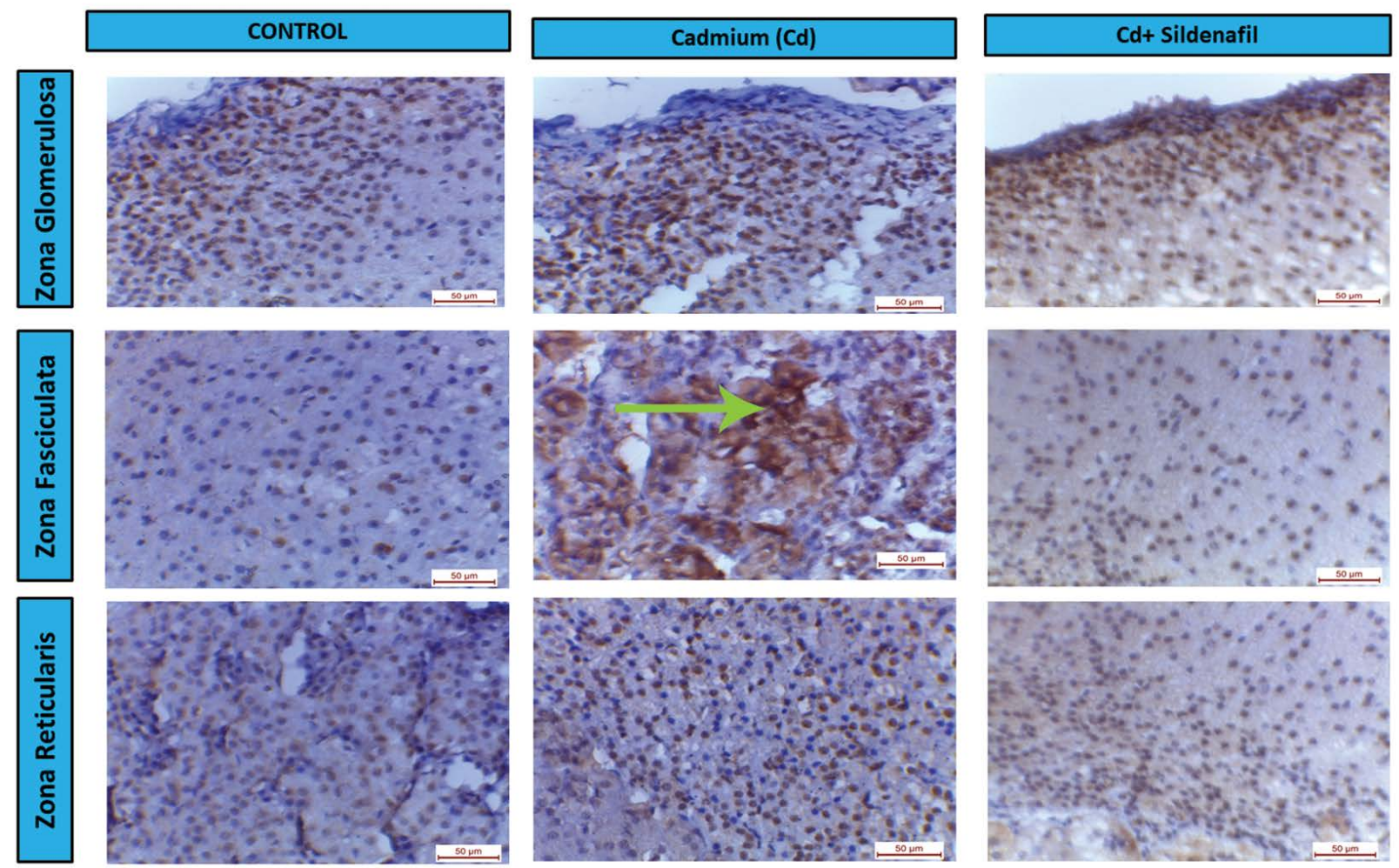

Figure 9. Cadmium group is showing increased caspase-3 (arrow) immunoexpression especially in Zona Fasciculata while cadmium + sildenafil group is showing decreased reaction (caspase- $3 \times 400$ ). 
while cadmium + sildenafil group showed moderate reaction.

By caspase-3 immunostaining (Fig. 9), control and sildenafil groups exhibited similar results with mild reaction in all zones of the cortex. Cadmium group exhibited increased caspase- 3 immunoexpression especially in ZF while cadmium + sildenafil group showed decreased reaction.

\section{Histomorphometric results}

The optical density of LAMP2, NF- $\kappa$ B and caspase-3 immunoexpression was significantly higher in cadmium group $(80 \%, 75 \%$, and $136 \%$, respectively) than control group. It was significantly lower $(31 \%, 32 \%$, and $35 \%$ ) in cadmium + sildenafil group than cadmium group. The optical density of LAMP2 and NF- $\kappa$ B immunoexpression was non-significantly higher $(19 \%, 18 \%$, respectively) in cadmium + sildenafil group than the control. The optical density of caspase-3 immunoexpression was significantly higher $(51 \%)$ in cadmium + sildenafil group than the control (Fig. 1).

\section{DISCUSSION}

Cadmium had induced adrenal cortical autophagy in the current work. Concomitant administration of sildenafil with cadmium had modulated this autophagy and other ultrastructural deleterious effects. The results of this study presented that exposure to cadmium had induced body weight loss in cd group despite no significant change in adrenal gland weight had been detected. Concordantly, Mutsuga et al. (2017) [21] had reported similar finding upon exposure to aminoglutathimide. The latter authors attributed this slight relative increase in the adrenal weight to lipid droplets accumulation. Adrenal gland MDA and SOD had been modulated by sildenafil as compared to cadmium received rats in the current work. This might indicate the antioxidative stress role of sildenafil. Supporting these findings, it was reported that sildenafil has significantly decreased the elevated MDA level in gentamicin-nephrotoxicity [20]. In addition, it was demonstrated that sildenafil had decreased renal MDA upon exposure to caecal ligation and puncture-induced sepsis in rats [5].

Frequent apoptotic cells were seen in cadmium group, while few apoptotic cells were found in cadmium + sildenafil group. This was confirmed by significant reduction in caspase- 3 immunoexpression in cadmium + sildenafil group. Supporting these results, sildenafil had been proved to ameliorate apoptosis in the ischaemic myocardium [7]. The mechanism by which sildenafil mitigates apoptosis has been suggested by some authors. Up-regulation of vascular endothelial growth factor expression [6] and promoting angiogenesis in the peri-infarct region after stroke $[9,17]$ had been proposed as mechanism of action. Opening of mitochondrial ATP-sensitive potassium (mito- $\mathrm{K}_{\text {ATP }}$ ) channels had been suggested to mediate the cardioprotective effects of sildenafil [7]. Also, activation of protein kinase $C$ and cGMP-dependent protein kinases [8] had been early proposed. However, the exact method by which sildenafil exerts as antiapoptotic is intriguing and warrants further researches.

In the present study, cadmium group showed increased vacuolation of the $Z G$ and ZF cells in $H \& E$ sections together with fatty degeneration in semithin sections. Fatty change, which may be reversible in moderate degrees, is accumulation of lipid droplets due to disruption of ribosomal function and uncoupling of lipid from protein metabolism [4]. Lipofuscin was detected in the current work in ZR of cadmium + sildenafil group. It is present normally in ZR of adrenal gland and is considered as an aging or atrophy insoluble brownish-yellow granular "wear-and-tear pigment" that accumulates in a variety of tissues representing complexes of lipid and protein that are produced by the free radical-catalysed peroxidation of polyunsaturated lipids of subcellular membranes [16, 19].

Adverse ultrastructural changes in the adrenals of cadmium group of this work were partially improved by sildenafil. Disruption of the mitochondrial cristae could be explained by accumulation of lipid granules caused by impaired steroidogenesis. The enzymes 11-hydroxylase (CYP11) in mitochondria and 17- and 21-hydroxylases (CYP 17 and 21) in the smooth endoplasmic reticulum had been suggested to be commonly disrupted by toxic chemicals [24]. The increased number of enlarged lysosomes containing degraded organelles in cadmium group and the significant increase in LAMP2 immunoexpression might indicate cadmium-induced autophagy in the adrenocortical cells. Although not measured in the present work, transmission electron microscopy morphometry for quantification of autophagy has been reported to be rather controversial, and unreliable procedures still continue to be used [14]. The relation between autophagy and cancer had been established. Cadmium-induced autophagy in the current work might suggest a clue to the link between cadmium and prostatic cancer [23] and further researches are 
still deserved. Whether autophagy might be good or bad from the vantage point of the tumour, however, stay a matter of debate and active investigation [16]. Modulation of LAMP2 immunoexpression by sildenafil in this work is concordant with its ultrastructural regression in the number of enlarged lysosomes induced by cadmium. LAMP2-positive granules had been reported by Mutsuga et al. (2017) [21] to be increased in the fasciculata cells of aminoglutethimide (antitumour agent in breast and prostate cancer) reflecting its-induced intracellular mitophagy and lipophagy, which are protein degradation processes for damaged mitochondria and excessively accumulated lipid droplets, respectively [26, 28].

$\mathrm{NF}-\kappa \mathrm{B}$ immunoexpression had been significantly increased in cadmium receiving rats. Sildenafil had modulated this expression. NF- $\kappa$ B had been considered as an important transcription factor, participates in growth, differentiation, organogenesis, apoptosis, inflammation and immune response [18]. The link between $\mathrm{NF}-\kappa \mathrm{B}$ and autophagy in the current work might have been established in cadmium group. Concordantly, quinazolinediamine which is an NF- $\kappa \mathrm{B}$ inhibitor had been proved to ameliorate both autophagy and apoptosis induced by brefeldin [31]. Agreeing with the results of this work regarding the relation between sildenafil and NF- $\kappa \mathrm{B}$, it had been proved that roflumilast, a phosphodiesterase- 4 inhibitor, might mitigate cadmium-induced renal toxicity via modulation of NF- $\kappa \mathrm{B}$ activation and induction of NQO1 in rats $[3,29]$.

The crosstalk between apoptosis and autophagy in cadmium-induced adrenal cortical injury had been demonstrated in the current work. This crosstalk might be mediated through caspase- 3 activation, impairment of antioxidant defence and NF- $\kappa \mathrm{B}$ activation as proved from immunohistochemical and biochemical results of the present work. The latter hypothesis was supported by an earlier work that suggested that this crosstalk was mediated via caspase cleavage of beclin 1 [10]. Contradictory to our hypothesis, autophagy had been proven to possess a pivotal role in neuronal antioxidant pathway [11]. Further mechanistic studies are still warranted to investigate this crosstalk.

Clinically implicated from the current work, cadmium might induce adrenal cortical autophagy and apoptosis. Sildenafil might be valuable in preventing such adverse changes. However, the link between cadmium-induced autophagy, risk of cancer and an- tioxidant defence system is intriguing and further researches are deserved.

\section{CONCLUSIONS}

Cadmium might induce adrenal cortical autophagy in rats and sildenafil might show an ameliorating effect probably through enhancement of antioxidant defence mechanism and modulation of NF- $\kappa \mathrm{B}$.

\section{REFERENCES}

1. Adeyanju AA, Molehinn OR, Ige ET, et al. Sildenafil, a phosphodiesterase-5-inhibitor decreased the oxidative stress induced by carbon tetrachloride in the rat kidney: A preliminary study. J Applied Pharmaceutical Scien. 2018, doi: 10.7324/japs.2018.8217.

2. Aktas $C$, Kanter M, Erboga M, et al. Anti-apoptotic effects of curcumin on cadmium-induced apoptosis in rat testes. Toxicol Ind Health. 2012; 28(2): 122-130, doi: 10.1177/0748233711407242, indexed in Pubmed: 21632575.

3. Ansari MN, Aloliet RI, Ganaie MA, et al. Roflumilast, a phosphodiesterase 4 inhibitor, attenuates cadmium-induced renal toxicity via modulation of NF- $\kappa$ B activation and induction of NQO1 in rats. Hum Exp Toxicol. 2019; 38(5): 588-597, doi: 10.1177/0960327119829521, indexed in Pubmed: 30744402.

4. Bury J. Responses to cellular injury, In: Cross SS, editor. 7th edition. Underwood's Pathology, Elsevier 2019: 77-94.

5. Cadirci E, Halici Z, Odabasoglu F, et al. Sildenafil treatment attenuates lung and kidney injury due to overproduction of oxidant activity in a rat model of sepsis: a biochemical and histopathological study. Clin Exp Immunol. 2011; 166(3): 374-384, doi: 10.1111/j.1365-2249.2011.04483.x, indexed in Pubmed: 22059996.

6. Caretti A, Bianciardi P, Ronchi R, et al. Phosphodiesterase- 5 inhibition abolishes neuron apoptosis induced by chronic hypoxia independently of hypoxia-inducible factor-1alpha signaling. Exp Biol Med (Maywood). 2008; 233(10): 1222-1230, doi: 10.3181/0802-RM-73, indexed in Pubmed: 18641057.

7. Das A, Durrant D, Salloum FN, et al. PDE5 inhibitors as therapeutics for heart disease, diabetes and cancer. Pharmacol Ther. 2015; 147: 12-21, doi: 10.1016/j.pharmthera.2014.10.003, indexed in Pubmed: 25444755.

8. Das A, Xi L, Kukreja RC. Protein kinase G-dependent cardioprotective mechanism of phosphodiesterase- 5 inhibition involves phosphorylation of ERK and GSK3beta. J Biol Chem. 2008; 283(43): 29572-29585, doi: 10.1074/jbc. M801547200, indexed in Pubmed: 18723505.

9. Ding G, Jiang Q, Li L, et al. Longitudinal magnetic resonance imaging of sildenafil treatment of embolic stroke in aged rats. Stroke. 2011; 42(12): 3537-3541, doi: 10.1161/ STROKEAHA.111.622092, indexed in Pubmed: 21903952.

10. Djavaheri-Mergny M, Maiuri MC, Kroemer G. Cross talk between apoptosis and autophagy by caspase-mediated cleavage of Beclin 1. Oncogene. 2010; 29(12): $1717-$ -1719, doi: 10.1038/onc.2009.519, indexed in Pubmed: 20101204 
11. Giordano S, Darley-Usmar V, Zhang J. Autophagy as an essential cellular antioxidant pathway in neurodegenerative disease. Redox Biol. 2014; 2: 82-90, doi: 10.1016/j. redox.2013.12.013, indexed in Pubmed: 24494187.

12. Khalaf HA, Ghoneim FM, Arafat EA, et al. Histological effect of nicotine on adrenal zona fasciculata and the effect of grape seed extract with or without withdrawal of nicotine. J Microsc Ultrastruct. 2017; 5(3): 123-131, doi: 10.1016/j. jmau.2016.11.001, indexed in Pubmed: 30023246.

13. Kierszenbaum A, Tres L. Endocrine system. Histology and Cell Biology: An Introduction to Pathology. Chapter 19. Saunders, an imprint of Elsevier Inc, Philadelphia. 2016: 561-581, doi: 10.1016/b978-0-323-07842-9.50023-x.

14. Klionsky DJ, Abdelmohsen K, Abe A, et al. Guidelines for the use and interpretation of assays for monitoring autophagy (3rd edition). Autophagy. 2016; 12(1): 1-222, doi: 10.1080/15548627.2015.1100356, indexed in Pubmed: 26799652.

15. Kumar V, Abbas AK, Aster JC. Neoplasia In: Robbins Basic Pathology, 10th edition. Chapter 6. Elsevier, Philadelphia 2018: 189-242.

16. Kumar V, Abbas AK, Aster JC. Cell Injury, Cell Death, and Adaptations. In: Robbins Basic Pathology, 10th edition. Chapter 2. Elsevier, Philadelphia 2018: 31-56.

17. Li L, Jiang $Q$, Zhang Li, et al. Angiogenesis and improved cerebral blood flow in the ischemic boundary area detected by MRI after administration of sildenafil to rats with embolic stroke. Brain Res. 2007; 1132(1): 185-192, doi: 10.1016/j. brainres.2006.10.098, indexed in Pubmed: 17188664.

18. Liang $Y$, Chen $G$, Yang $Y$, et al. Effect of canonical NF- $\kappa$ B signaling pathway on the differentiation of rat dental epithelial stem cells. Stem Cell Res Ther. 2019; 10(1): 139, doi: 10.1186/ s13287-019-1252-7, indexed in Pubmed: 31109359.

19. Lowe JS, Anderson PG, Anderson SI. The Cell. In: Stevens \& Lowe's Human Histology, 5th edition. Chapter 2. Elsevier, Philadelphia 2020: 12-40.

20. Morsy MA, Ibrahim SA, Amin EF, et al. Sildenafil ameliorates gentamicin-induced nephrotoxicity in rats: role of iNOS and eNOS. J Toxicol. 2014; 2014: 489382, doi: 10.1155/2014/489382, indexed in Pubmed: 25120567.

21. Mutsuga $M$, Asaoka $Y$, Imura N, et al. Aminoglutethimide-induced lysosomal changes in adrenal gland in mice. Exp Toxicol Pathol. 2017; 69(7): 424-429, doi: 10.1016/j. etp.2017.04.004, indexed in Pubmed: 28410883.
22. Orororo OC, Asagba SO, Tonukari NJ, et al. Effects of Hibiscus Sabdarrifa L. anthocyanins on cadmium-induced oxidative stress in Wistar rats. J Appl Sci Environ Manag. 2018; 22(4): 465, doi: 10.4314/jasem.v22i4.4.

23. Rapisarda V, Miozzi E, Loreto $C$, et al. Cadmium exposure and prostate cancer: insights, mechanisms and perspectives. Front Biosci (Landmark Ed). 2018; 23: 1687-1700, doi: 10.2741/4667, indexed in Pubmed: 29293457.

24. Rosol TJ, Yarrington JT, Latendresse J, et al. Adrenal gland: structure, function, and mechanisms of toxicity. Toxicol Pathol. 2001; 29(1): 41-48, doi: 10.1080/019262301301418847, indexed in Pubmed: 11215683.

25. Sanderson S, Wild G, Cull AM, Marston J, Zardin G. Immunohistochemical and immunofluorescent techniques. In: Suvarna SK, Layton C, Bancroft, JD, Editors. Bancroft's Theory and Practice of Histological Techniques, Eighth Edition, Elsevier Limited 2019: 337-394.

26. Singh R, Cuervo AM. Lipophagy: connecting autophagy and lipid metabolism. Int J Cell Biol. 2012; 2012: 282041, doi: 10.1155/2012/282041, indexed in Pubmed: 22536247.

27. Tulis DA, Middlemas DS. Vasodilators for Hypertensive Crises, Pulmonary Hypertension, and Erectile Dysfunction. In Brody's Human Pharmacology, Wecker L, Taylor DA, Theobald RJ. Elsevier, Kirksville, Missouri. 6th edition 2019: 330-336.

28. Williams JA, Ding WX. A mechanistic review of mitophagy and its role in protection against alcoholic liver disease. Biomolecules. 2015; 5(4): 2619-2642, doi: 10.3390/ biom5042619, indexed in Pubmed: 26501336.

29. Yuan G, Dai S, Yin Z, et al. Sub-chronic lead and cadmium co-induce apoptosis protein expression in liver and kidney of rats. Int J Clin Exp Pathol. 2014; 7: 2905-2914, indexed in Pubmed: 25031709.

30. Zhang R, Wang Y, Zhang Li, et al. Sildenafil (Viagra) induces neurogenesis and promotes functional recovery after stroke in rats. Stroke. 2002; 33(11): 2675-2680, doi: 10.1161/01.str.0000034399.95249.59, indexed in Pubmed: 12411660.

31. Zhu X, Huang Li, Gong J, et al. NF-B pathway link with ER stress-induced autophagy and apoptosis in cervical tumor cells. Cell Death Discov. 2017; 3: 17059, doi: 10.1038/cddiscovery.2017.59, indexed in Pubmed: 28904818. 